\title{
JEAN DE WAVRIN, UN CHRONIQUEUR ENTRE BOURGOGNE ET ANGLETERRE, ET SES HOMOLOGUES BOURGUIGNONS FACE À LA GUERRE DES DEUX ROSES
}

\section{Alain Marchandisse}

De Boeck Supérieur | « Le Moyen Age »

2006/3 Tome CXII | pages 507 à 527

ISSN 0027-2841

ISBN 2-8041-5168-9

Article disponible en ligne à l'adresse :

https://www.cairn.info/revue-le-moyen-age-2006-3-page-507.htm

\section{Pour citer cet article :}

Alain Marchandisse, " Jean de Wavrin, un chroniqueur entre Bourgogne et Angleterre, et ses homologues bourguignons face à la guerre des Deux Roses », Le Moyen Age 2006/3 (Tome CXII), p. 507-527.

DOI 10.3917/rma.123.0507

Distribution électronique Cairn.info pour De Boeck Supérieur.

(C) De Boeck Supérieur. Tous droits réservés pour tous pays.

La reproduction ou représentation de cet article, notamment par photocopie, n'est autorisée que dans les limites des conditions générales d'utilisation du site ou, le cas échéant, des conditions générales de la licence souscrite par votre établissement. Toute autre reproduction ou représentation, en tout ou partie, sous quelque forme et de quelque manière que ce soit, est interdite sauf accord préalable et écrit de l'éditeur, en dehors des cas prévus par la législation en vigueur en France. Il est précisé que son stockage dans une base de données est également interdit. 


\section{Jean de Wavrin, un chroniqueur entre Bourgogne et Angleterre, et ses homologues bourguignons face à la guerre des Deux Roses ${ }^{1}$}

Force est de le constater : si plusieurs écrivains du $X V^{e}$ siècle, bourguignons ou apparentés - les Chastelain, Commynes, La Marche et autre Molinet - se sont vu consacrer pléthore de livres, d'articles et de contributions de qualité, pour d'autres - d'Escouchy, Du Clercq ou Lefèvre de Saint-Rémy, pour ne citer que les plus connus -, la terra n'est pas loin d'être encore obstinément incognita ${ }^{2}$. De Jean de Wavrin, dont il sera principalement question ici, l'on peut dire qu'il se situe en quelque sorte au milieu du gué. Son Recueil des croniques et anciennes istoires de la Grant Bretaigne, a presant nommé Engleterre a été édité à deux reprises. Il y a à peu près un siècle et demi, E. Dupont proposait un texte s'étendant de 1325 à 1471, mais l'amputait des passages qu'elle jugeait empruntés aux devanciers de Wavrin et des récits consacrés

1. Pour l'élaboration de cette étude, j'ai pu bénéficier de l'appui bibliographique et technique de Madame L. Visser-Fuchs ainsi que de mes collègues et amis Messieurs Ph. Demonty (ULg, Commission royale d'Histoire), J. Devaux (Université du Littoral-Côte d'Opale, Dunkerque), J.Fr. NiEus (F.N.R.S./F.U.N.D.P, Namur) et H. WiJsman (Université de Leyde). Qu'ils en soient chaleureusement remerciés. Pour connaître l'expression complète des titres abrégés tout au long de ce travail, le lecteur voudra bien se reporter à la bibliographie thématique du sujet traité, située à la fin de la section renfermant les présents actes des rencontres de Dunkerque consacrées aux Littérature et culture historiques à la cour de Bourgogne.

2. Cf. Bibliographie thématique, section Travaux critiques, tout spécialement pour les premiers écrivains mentionnés, les diverses études des auteurs suivants : SMALL, Delclos, Doudet, Dufournet, Blanchard, Emerson et Devaux. 
à l'Angleterre ; elle conservait uniquement la matière focalisée sur le continent et en particulier sur la Bourgogne ${ }^{3}$. À peu près à la même époque, tout au moins pour le premier des cinq volumes publiés, W. Hardy consacrait son édition à la période comprise entre la naissance du royaume d'Albion et 688,

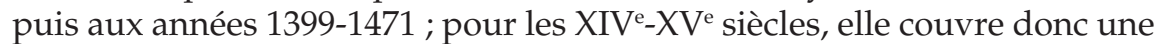
période plus restreinte que celle d'E. Dupont, mais présente l'avantage péremptoire de ne pas avoir été caviardée ${ }^{4}$. Même si elle n'a pas passionné la foule des chercheurs, $l^{\prime} œ u v r e$ de Wavrin a fait l'objet de quelques travaux, depuis celui, déjà ancien, de J. Stengers, qui s'intéressait aux relations mutuelles entre Wavrin et d'autres auteurs ${ }^{5}$, jusqu'aux quelques pages consacrées au chroniqueur par $\mathrm{M}$. Zingel dans un ouvrage trop rarement cité ${ }^{6}$, et à $l^{\prime}$ article, très récent, de G. Le Brusque, voué au récit que fait Wavrin de l'expédition de son neveu, Waleran, contre les Turcs ottomans, en 1444$1446^{7}$, en passant par les diverses synthèses d'A. Naber portant sur la passion des livres dont fit montre le chroniqueur ${ }^{8}$. Il n'en reste pas moins que Jean de Wavrin n'a pas encore rencontré son J. Dufournet, sa C. Emerson ou son J. Devaux, même si, comme pouvait déjà le laisser présager la publication d'un certain nombre d'études ${ }^{9}$, principalement centrées sur l'Angleterre, par L. Visser-Fuchs, cette dernière annonce la prochaine sortie de presse d'un livre sur le chroniqueur, issu de sa thèse de doctorat défendue en 2002 à $l^{\prime}$ Université de Londres ${ }^{10}$. Sans prétendre à une parfaite originalité, j'ai cru

3. J. DE WAVRIN, Anchiennes chronicques d'Engleterre, éd. Dupont, ici t. 1, p. I-II.

4. ID., Recueil des croniques et anchiennes istories de la Grant Bretaigne, éd. HARDY.

5. Stengers, Sur trois chroniqueurs.

6. ZINGEL, Frankreich, das Reich und Burgund, p. 70-88.

7. Le BRUSQUE, Des chevaliers bourguignons dans les pays du Levant (et bibl.). Cf. encore, en dernier lieu sans doute: C. IMBER, The Crusade of Varna, 1443-45, Aldershot, 2006, où l'événement se trouve relaté par des extraits de sources diverses en traduction, dont la chronique de Jean de Wavrin.

8. NABER, Jean de Wavrin bibliophile ; ID., Manuscrits d'un bibliophile bourguignon ; ID., Les goûts littéraires.

9. Notamment VISSER-FuCHS, Edward IV's «memoir on paper »; ID., Jean de Wavrin and the foundation of Britain; ID., Jean de Wavrin and the English newsletters ; ID., Pour recreer les esperitz ; ID., « Warwick, by himself ». Certaines des questions simplement évoquées ici y trouvent d'amples, subtils et pertinents développements.

10. Titre: Warwick and Wavrin. Two case studies on the literary background and propaganda of Anglo-Burgundian relations in the Yorkist period. Il n'est pas inutile de préciser qu'aucune des études de L. Visser-Fuchs, où, parfois, il est fait référence à Wavrin jusque dans le titre, n'est mentionnée dans la Bibliographie der französischen Literatur-Wissenschaft de O. KLAPp, t. 1 et s., Francfort, 1960-. L'on ajoutera encore à la bibl. : G. TYL-LaborY, Art. Jean de Wavrin, Dictionnaire des Lettres françaises. Le Moyen Âge, éd. G. HasenoHr et M. Zink, Paris, 1992, p. 861-862 ; M. YANS, Art. Wavrin ou Waurin (Jean de), Biographie nationale de Belgique, t. 27, Bruxelles, 1938, col. 129-132 ; A. Gransden, Historical Writing in England, t. 2, C. 1307 to the Early Sixteenth Century, Londres-New York, 1996, p. 288-293. 
bon de mener ma recherche consacrée à Wavrin dans deux directions complémentaires. Dans un premier temps, je me pencherai sur la causa scribendi de ce chroniqueur médiéval, moins sans doute sur les raisons qui l'ont conduit à entreprendre un «travail d'historien » et à élaborer une histoire très largement britannique, que sur celles qui, peut-être - cela reste une simple hypothèse de travail -, expliquent comment et pourquoi cet auteur, à la fin de sa vie, dans les derniers livres de son œuvre et dans la probable révision de ces derniers, a manifestement conçu une considération toute particulière, qui est le reflet d'un choix personnel et pas seulement des options politiques bourguignonnes du moment, pour les plus hautes sphères de la cour yorkiste. Dans une seconde phase, je m'intéresserai, davantage pour la trame que pour le détail des faits, à la narration que proposent Wavrin et ses principaux homologues, contemporains et successeurs, à ce qu'ils en savent et à ce qu'ils en livrent à leur public et, in fine - là réside mon principal dessein -, à la connaissance et la perception que put avoir la société bourguignonne de l'époque de Wavrin d'un conflit fameux entre tous, qui battait alors son plein Outre-Manche : la guerre des Deux Roses.

Avant que d'en venir à cette matière, sans doute n'est-il pas inutile que l'on en jette les premiers jalons en retraçant quelque peu la vie et l'œuvre de Wavrin. Né aux environs de 1400, il est le fils naturel de Robert VII, seigneur de Wavrin, sénéchal héréditaire de Flandre, conseiller-chambellan du duc de Bourgogne, un seigneur originaire de la région lilloise dont le lignage fit florès durant les XII ${ }^{\mathrm{e}}$ et XIII ${ }^{\mathrm{e}}$ siècles, à la cour comtale de Flandre. Celui qui est souvent appelé le Bâtard de Wavrin est présent à Azincourt, peut-être comme officier d'armes, et y débute dans la carrière militaire. Il y rencontre son futur collègue chroniqueur, Jean Lefèvre de Saint-Rémy. En 1417, il est écuyer au sein d'une compagnie de Philippe de Saveuses, alors que celui-ci en fait la montre devant un Jean sans Peur décidé à marcher sur Paris, et appartient donc bien au parti bourguignon, puis, après peut-être un séjour en Terre sainte en 1418, participe en 1421 à la deuxième croisade contre Prague et les Hussites. Les années 1423-1437 ne seront pour lui qu'expéditions militaires, en 1427, par exemple, dans l'armée du duc de Bourgogne Philippe le Bon, alors aux prises avec la comtesse de Hainaut Jacqueline de Bavière, puis dans les rangs anglais, dès 1423, avant le siège de Calais, possession anglaise, auquel le contraint, en 1436, la nouvelle orientation de la politique bourguignonne. L'année 1437 est capitale pour Wavrin : il quitte l'armée, se marie avec une veuve passablement argentée de la bourgeoisie lilloise, est légitimé par le duc de Bourgogne et se voit rétribué pour ses prestations armées, mais n'en continue pas moins à le servir. En 1442, il apparaît comme chevalier, détenteur de diverses seigneuries, plus tard, dans les années soixante, comme conseiller et chambellan du duc, après une nouvelle légitimation en 1447, des œuvres cette fois du roi de France Charles 
VII. En 1463, il fait partie de l'ambassade envoyée auprès du pape Pie II afin de discuter de l'éternelle croisade contre les Turcs, puis, en 1464, d'une délégation ducale auprès des états de Hainaut, où l'on doit prestement évoquer la menace que représente alors la ville de Liège. En 1467, il accompagne le Grand bâtard, Antoine de Bourgogne, à la cour d'Angleterre, tandis qu'en 1469, il est le témoin de la rencontre, à Saint-Omer, entre Richard Neville, comte de Warwick et de Salisbury, le célèbre « Faiseur de roi », et le Hardi-Téméraire. L'on situe sa mort dans les années suivant 1471, entre 1472 et 1475 . Jean de Wavrin passe pour être l'auteur de plusieurs œuvres littéraires. Quoi qu'il en ait été, il se constitua une bibliothèque très riche et son nom reste attaché au maître miniaturiste dit de Wavrin et à l'atelier qui enluminèrent la majeure partie des douze manuscrits, principalement des romans de chevalerie, qu'il commandita ${ }^{11}$.

Venons-en à présent à la rédaction de l'œuvre majeure du bâtard de Wavrin, son Recueil des chroniques et histoires d'Angleterre, et à sa chronologie. Sans s'être engagés dans une quelconque polémique, les éditeurs et commentateurs de l'œuvre de Wavrin ne semblent pas présenter des visions rigoureusement convergentes quant aux diverses étapes de sa composition. Voici ce qui paraît pouvoir être dit à ce propos sans que cela ne suscite aussitôt une levée de boucliers ${ }^{12}$. Une première mouture de l'œuvre paraît avoir été entamée en 1446.

À s'en remettre au témoignage du chroniqueur, ce dernier aurait entrepris la chronique grâce à laquelle son nom a survécu à l'oubli à l'instigation de son neveu, Waleran, seigneur de Wavrin, le fils de sa sœur Béatrice, celui-là même que le duc de Bourgogne Philippe le Bon avait nommé lieutenant et

11. Tyl-LabOrY, Art. Jean de Wavrin ; Yans, Art. Wavrin ou Waurin (Jean de) ; NABER, Jean de Wavrin bibliophile ; ZINGEL, Frankreich, das Reich und Burgund, p. 7071 ; Gransden, Historical Writing in England, t. 2, p. 288-289 ; J. DE Wavrin, Anchiennes chronicques d'Engleterre, éd. Dupont, t. 1, p. XIII-XXXI ; ID., Recueil des croniques et anchiennes istories de la Grant Bretaigne, éd. HARDY, t. 1, p. XVII-XLVI. Sur le maître de Wavrin - et sur la bibliothèque du Bâtard, d'ailleurs -, voir en dernier lieu P. Schandel, Histoire des seigneurs de Gavre, Art de l'enluminure, fasc. 3, s. d., et bibl. p. 16, en particulier sa thèse de doctorat inédite: ID., Le Maître de Wavrin et les miniaturistes lillois à l'époque de Philippe le Bon et de Charles le Téméraire, Strasbourg, Université Marc Bloch Strasbourg 2, 1997. Wavrin (France, Nord), Lillers (Pas-deCalais).

12. J. De Wavrin, Anchiennes chronicques d'Engleterre, éd. Dupont, t. 1, p. XXXIXXXVI ; ID., Recueil des croniques et anchiennes istories de la Grant Bretaigne, éd. HARDY, t. 1, p. XLII-XLIX; GransDen, Historical Writing in England, t. 2, p. 264, 289-290 ; ZINGEL, Frankreich, das Reich und Burgund, p. 71-75 ; TYL-LABORY, Art. Jean de Wavrin ; NABER, Jean de Wavrin bibliophile, p. 288 ; ID., Manuscrits d'un bibliophile bourguignon, p. 23. 
capitaine general ${ }^{13} \mathrm{~d}^{\prime} \mathrm{un}$ ensemble de navires chargés d'apporter un appui militaire à l'empereur de Constantinople Jean VIII Paléologue dans sa lutte contre l'Infidèle. Ainsi lui rend-il hommage au cœur de son prologue : par pluiseurs fois vous pleut prendre voz devises a moy, touchans de pluiseurs belles et anchiennes hystoires entre lesquelles encommenciastes a parler de ce tresnoble et anchien royaulme de la Grant Bretaigne. [...] aiant le bon voloir de vostre noble desir, moiennant davoir vostre bonne ayde et conseil, qui a ceste matere m'a este bien seant, ay oze entreprendre ceste paine et labeur ${ }^{14}$. L'on peut penser que Jean de Wavrin, sachant son neveu encore très au fait des détails de son expédition orientale, commence par rédiger le récit de ses exploits - il s'agit de ce que l'historiographie anglo-saxonne nomme « newsletters », que l'on peut définir comme des «narratives written to describe an event with the express purpose of recording it for the benefit of people who had not been present $t^{15}$ », puis entreprend de rassembler tout un ensemble d'informations qu'il met en forme une première fois en 1455. Répartie sur quatre volumes divisés, chacun, en six livres, cette Histoire d'Angleterre couvre alors la période allant des origines légendaires du pays jusqu'à la mort d'Henri IV de Lancastre, en 1413. Ultérieurement, deux volumes supplémentaires, soumis à une même partition, seront élaborés, le cinquième portant la matière jusqu'en 1443, le sixième jusqu'à la seconde montée d'Édouard IV d'York sur le trône d'Angleterre, en 1471. Il est délicat de se prononcer sur la date à laquelle chacun de ces deux derniers volumes a été réalisé. Pour le cinquième, l'on a évoqué des prémisses après 1455 ou mis en exergue l'année 1461 et la première accession à la couronne du même Édouard IV, précisant que le volume était encore loin d'être achevé à cette date. Du sixième volume, l'on peut au moins dire qu'il est contemporain des derniers événements qu'il relate ; le point final ne peut évidemment avoir été mis avant 1471 , date très proche de celle du décès de l'auteur. Toutefois, selon M. Zingel, Wavrin utilisa un texte anglais intitulé The Historie of the Arrival of King Edward IV A.D. 1471 , lequel fut réalisé d'après une version courte en langue française datant d'entre mai 1471 et mars 1472 : le volume six de son Recueil ne peut avoir été

13. Le Brusque, Des chevaliers bourguignons dans les pays du Levant, p. 257 et n. 8. Sur Waleran de Wavrin, cf. ZINGEL, Frankreich, das Reich und Burgund, p. 71 et $\mathrm{n}$. 17 (bibl.) et J. PAviot, Art. W., Waleran de, Lexikon des Mittelalters, rééd., t. 8, Munich, 2003, col. 2081-2082 (et bibl.), auxquels j'ajouterai volontiers ses deux ouvrages, où il est largement question du personnage et de l'œuvre du Bâtard, qui permettent de le mieux connaître : ID., La politique navale des ducs de Bourgogne, 1384/1482, Lille, 1995, spéc. les p. 113-123 ; ID., Les ducs de Bourgogne, la croisade et l'Orient (fin XIVe siècle-XVe siècle), Paris, 2003, spéc. les p. 97-108.

14. Les deux citations dans J. DE WAVRIN, Recueil des croniques et anchiennes istories de la Grant Bretaigne, éd. HARDY, t. 1, p. 2-3.

15. À ce propos, voir VISSER-FUCHS, Edward IV's « memoir on paper » ; ID., Jean de Wavrin and the English newsletters, cit. p. 218. 
achevé avant la seconde de ces dates, voire plus tard encore ${ }^{16}$. L'on sait cependant qu'en 1469, à l'issue de la rencontre à Saint-Omer entre Charles de Bourgogne et Warwick, Wavrin, desirant scavoir et avoir matieres veritables pour le parfait de [son] euvre, rejoignit le second à Calais, ce dernier lui ayant promis qu'il lui bailleroit homme qui [l']adrescheroit a tout ce que [il] vouldroi[t] demander touchantces matieres ${ }^{17}$. Wavrin fera globalement chou blanc, car Warwickétait alors préoccupé par les noces de sa fille avec Georges, duc de Clarence, le frère d'Édouard IV. Reste qu'il travaillait à son dernier livre en 1469 et ce millésime, qui est d'ailleurs celui privilégié par G. Tyl-Labory dans l'article qu'elle consacra à Wavrin dans le Dictionnaire des Lettres françaises ${ }^{18}$, pourrait bien être une date clé dans la mise en œuvre du sixième volume de cette Histoire d'Angleterre et dans la révision de celle-ci, qui semble assurée. L'on ajoutera enfin que, dans l'exemplaire du Recueil que Wavrin choisit de dédier à Édouard IV, à savoir les manuscrits Royal. 14. et 15.E.IV de la British Library, le prologue du volume un évoque le souhait del'auteur de composer un septième volume, consacré aux hauts faits du roi consécutifs à son retour aux affaires, en $1471^{19}$.

Quelles sont les raisons pour lesquelles Wavrin entreprit de composer une chronique et pourquoi choisit-il de la consacrer à l'Angleterre ? On l'a dit, la vocation littéraire du bâtard de Wavrin doit beaucoup à son neveu, Waleran, qui l'incita à retracer une histoire que nul clerc ne s'était jamais avisé jusqu'alors de peindre, si ce n'est sous la forme de biographies, celle des rois et des princes anglais. Mais, chez Wavrin, l'attrait pour l'Angleterre n'est pas seulement de prédilection intellectuelle. Il est certes anglophile ; il est plus encore et surtout, politiquement et militairement, un partisan convaincu d'une Angleterre dont les ducs de Bourgogne se firent un temps une alliée. En 1423, l'on trouve Wavrin à la bataille de Cravant, qui vit la défaite du roi de France Charles VII et de celui qui est appelé le connétable de l'armée d'Écosse, Jean Stuart, duc de Darnley, sous les coups du maréchal de Bourgogne Jean, seigneur de Toulongeon, et des siens, aidés par des forces anglaises commandées par les comtes de Salisbury et de Suffolk, Thomas Montagu et William de la Pole, dans les armées desquels Wavrin combattra.

16. ZINGEL, Frankreich, das Reich und Burgund, p. 73 n. 33.

17. J. DE WAVRIN, Recueil des croniques et anchiennes istories de la Grant Bretaigne, éd. HARDY, t. 5, p. 578-579.

18. TYL-Labory, Art. Jean de Wavrin, p. 861.

19. J. DE WAVRIn, Anchiennes chronicques d'Engleterre, éd. Dupont, t. 1, p. XLVIXLVIII ; ID., Recueil des croniques et anchiennes istories de la Grant Bretaigne, éd. HARDY, t. 1, p. 608-611. Cf. M. KerewICH, Edward IV, William Caxton, and literary Patronage in Yorkist England, The modern Language Review, t. 66, 1971, p. 484-485 ; J. DE WAVRIN, Anchiennes chronicques d'Engleterre, éd. Dupont, t. 1, p. III, VIII-X n. ; ID., Recueil des croniques et anchiennes istories de la Grant Bretaigne, éd. HARDY, t. 1, p. CCXIII-CCXV. 
En 1424, il assiste à la reddition du château d'Ivry-la-Bataille, alors dauphinois, en compagnie du duc de Bedford, puis à la bataille de Verneuil-surAvre, qui constitue une nouvelle défaite française. En 1429, on le retrouve, désormais pleinement au service d'Henri VI d'Angleterre et de Bedford, à Beaugency et à Patay, qui, cette fois, sont autant de victoires françaises et de cruelles désillusions pour les chefs anglais John Talbot et John Fastolf. Avec le traité d'Arras de 1435, la bonne entente qui prévalait jusqu'alors entre Bourguignons et Anglais se conjugua désormais au passé et nul doute que cette nouvelle donne devait finir par mettre Wavrin dans un réel embarras, pour peu qu'il soit amené à rencontrer ses amis d'hier sur un champ de bataille. Ce n'est sans doute pas indifférent si c'est peu avant qu'il ne commence à composer son œuvre qu'il abandonne le service militaire actif du duc de Bourgogne. Pour échapper à des options politiques qu'il ne partageait plus guère, Wavrin, qui ne souhaite pas non plus s'acagnarder, deviendra donc écrivain. Le parallèle avec l'écrivain Guillebert de Lannoy, qui lui aussi quitte l'armée au moment de la rupture entre la Bourgogne et l'Angleterre et se met à écrire, entre 1439 et 1445, une Instruction d'un jeune prince qui a pu passer pour une critique insidieuse d'Arras, ne manque $\mathrm{d}^{\prime}$ ailleurs pas d'être éclairant sur ce point ${ }^{20}$.

Au-delà des politiques menées par Édouard IV et Charles de Bourgogne, globalement convergentes jusqu'en août 1475, date à laquelle le souverain anglais signe le traité de Picquigny avec Louis XI, l'on doit fatalement se demander pourquoi le chroniqueur bourguignon, qui pourtant servit un temps dans les armées Lancastre et, même après Arras, ne peut s'empêcher de se montrer indulgent avec ceux qui, entre-temps, tel Humphrey de Gloucester, le frère de feu le roi Henri $V^{21}$, étaient, de par leur nationalité, devenus les ennemis des Bourguignons, fait désormais montre d'une réelle prédilection pour les York et va jusqu'à souhaiter dédicacer un exemplaire

20. Sur ces événements, cf. bibl. à la n. 11 ; J. DE WAVRIN, Anchiennes chronicques d'Engleterre, éd. Dupont, t. 1, p. XXIV-XXX. Sur les batailles en question, voir B. Schnerb, Art. Cravant (bataille de) et Ph. Contamine, Art. Verneuil-sur-Avre (bataille de) et Art. Patay (bataille de), dans le récent et très utile-bien que dépourvu de bibliographie - Dictionnaire Perrin des guerres et des batailles de l'histoire de France, sous la dir. de J. GARNIER, Paris, 2004, p. 248, 856, 650-651. L'on est certain que Wavrin et les frères Hugues et Guillebert de Lannoy se sont rencontrés, ces derniers ayant transmis au chroniqueur des informations dont il fera usage dans sa chronique (J. DE WAVRIN, Recueil des croniques et anchiennes istories de la Grant Bretaigne, éd. HARDY, t. 2, p. 229). Pour les réflexions relatives à Guillebert de Lannoy, cf. tout spécialement NABER, Jean de Wavrin bibliophile, p. 288. Cravant (France, Yonne), Ivry-la-Bataille et Verneuil-sur-Avre (Id., Eure), Beaugency et Patay (Id., Loiret).

21. Ibid., t. 4, p. 200-202. 
de son œuvre à Édouard IV. Ch.A.J. Armstrong a bien montré22 que l'excellente entente entre les cours yorkiste et bourguignonne tint pour beaucoup à la fortune grandissante, dans l'entourage du roi, d'une famille qui avait d'abord soutenu les Lancastre avant de recevoir le pardon des York: les Woodville. Sans en avoir informé le roi Henri VI, Richard Woodville, premier comte Rivers, avait épousé (1437) une personnalité importante de la noblesse « bourguignonne ", Jacqueline ou Jacquette de Luxembourg, veuve du duc de Bedford, fille du comte Pierre de Saint-Pol et sœur du célèbre connétable, et c'est tout aussi subrepticement que le fruit de cette mésalliance, Élisabeth, s'unit à Édouard IV (1464), sur un coup de tête amoureux pour celui-ci, de façon très calculée pour la reine. Cette décision sema la consternation parmi les barons anglais, et ce d'autant plus que, là où Warwick escomptait une alliance franco-anglaise, les Woodville allaient se déclarer très favorables au parti bourguignon et tout faire pour instiller semblable opinion dans l'esprit du $\mathrm{roi}^{23}$. L'on ne s'est peut-être pas suffisamment rendu compte qu'il est peut-être un Anglais, membre du lignage Woodville qui, plus que tout autre, pouvait avoir servi de trait d'union, voire de truchement entre le souverain anglais et un Jean de Wavrin qui finit par devenir son thuriféraire. Il s'agit du fils et du frère des Woodville évoqués plus haut, Anthony (ca 1440-1483), Lord Scales grâce à son mariage avec

22. Ch.A.J. Armstrong, L'échange culturel entre les cours d'Angleterre et de Bourgogne à l'époque de Charles le Téméraire, dans ID., England, France and Burgundy in the Fifteenth Century, Londres, 1982, p. 403-417, spéc. p. 404-410 (réimpr. de 1'art. paru dans Cinq-centième anniversaire de la bataille de Nancy (1477), p. 35-49).

23. Sur les Woodville, le mariage d'Édouard IV et d'Élisabeth, cf. M.A. Hicks, The Changing Role of the Wydevilles in Yorkist Politics to 1483, Patronage, Pedigree and Power in Later Medieval England, éd. Ch. Ross, Rowman-Littlefield, 1979, p. 60-86 (réimpr. dans ID., Richard III and his Rivals. Magnates and their Motives in the Wars of the Roses, Londres-Rio Grande, 1991, p. 209-228) et, notamment pour la période qui suit les propos égrainés par Wavrin : D.E. LowE, Patronage and Politics : Edward IV, the Wydevills, and the Council of the Prince of Wales, 1471-1483, Bulletin of the Board of celtic Studies, t. 29, 1981, p. 545-573, spécialement p. 553-556-et les pages y consacrées dans les diverses biographies respectives des deux personnages : C.L. SCOFIELD, The life and reign of Edward the Fourth, King of England and of France and Lord of Ireland, t. 1, Londres-New York-Toronto-Bombay-Calcutta-Madras, 1923, p. 332-333 ; Ch. Ross, Edward IV, Berkeley-Los Angeles, 1974, p. 84-103 ; M. НІскS, Edward IV, Londres, 2004, p. 107-114, 128-129, 208-209 ; D. McGIBBON, Elizabeth Woodville (1437-1492). Her Life and Times, Londres, 1938, p. 28-39 ; D. BALDwIN, Elizabeth Woodville, Mother of the Princes in the Tower, Phoenix Mill, Thrupp, Stroud, 2002, p. 11-13. À ces deux derniers ouvrages, je préfère M. Hicks, Art. Elizabeth [née Elizabeth Woodville], Oxford Dictionary of National Biography, t. 18, Oxford-New York, 2004, p. 79 (et bibl.). Cf. aussi ID., Art. Woodville [Wydeville], Richard, first Earl Rivers, Oxford Dictionary of National Biography, t. 60, p. 227-228. 
Élisabeth Scales, puis second comte Rivers au décès de son père en $1469^{24}$. Sorte de chevalier errant, passionné par les joutes, les croisades et les pèlerinages, Anthony Woodville était avant tout un professionnel des armes et appartenait à la fleur chevaleresque de la cour des York. En 1468, on le rencontre comme commandant d'une force navale destinée à soutenir la Bretagne contre la France; il interviendra à plusieurs reprises contre Warwick, contribuera à l'invasion anglaise de la France en 1475 et sera présent à Morat, avant d'être l'un des principaux chefs de l'envahissement de l'Écosse en 1482. Bien évidemment - et il en ira de même pour l'ensemble du lignage Woodville -, son existence sera bouleversée par l'accession au trône de sa sœur Élisabeth en 1464. Charges et titres s'amoncellent alors dans son escarcelle. Mais la puissance York s'étiole et Warwick tient Anthony pour un ennemi privilégié ; il accompagnera Édouard IV dans son exil, mais sera aussi l'un des artisans de sa seconde montée sur le trône d'Angleterre en 1471. Rivers décide alors de se consacrer à sa vie spirituelle et de partir en croisade, ce qui ne plaît pas nécessairement à son roi, qui a besoin de ses compétences. On le retrouve tout de même à la cour de Bourgogne en 14731474, où il est venu en ambassade. En 1477, Édouard IV lui destine pour seconde épouse rien moins que Marie de Bourgogne, l'héritière de Charles le Hardi, mais ni cette union, ni une autre, avec la sœur de Jacques III $\mathrm{d}$ 'Écosse, n'aboutiront. La suite de sa carrière importe peu ici. L'on retiendra simplement qu'il fut le mentor et l'un des soutiens politiques du prince Édouard de Galles, le futur Édouard V, et qu'il sera exécuté le 25 juin 1483, sur ordre de celui qui, le jour suivant, allait devenir le roi Richard III d'Angleterre.

Deux faits majeurs de la vie d'Anthony Woodville semblent de nature à avoir fait de lui un bon agent de liaison entre le souverain anglais et ce représentant éminent de la culture bourguignonne qu'était Wavrin. L'on se doit tout d'abord d'insister sur le fait qu'Anthony eut une véritable carrière littéraire, d'ailleurs en rapport avec son goût ostentatoire du religieux. L'on connaît en effet, grâce à William Caxton, traducteur, éditeur, fondateur de l'imprimerie en Angleterre († 1492), l'attrait de Rivers pour les œuvres de piété et son dégoût pour le péché, des choix de vie qui semblent l'avoir conduit à traduire en anglais, sous les titres The Dictes and Sayings of the Philosophers, The Moral Proverbs et The Cordyal, des œuvres en langue étrangère: les Dits moraux des philosophes, traduits en prose, du latin, par Guillaume de Tignonville (avant 1402), les Proverbes moraux de Christine de

24. Une biographie de ce personnage important et singulier manque. Outre la bibl. mentionnée dans les n. précédentes, notamment les études de Ch.A.J. Armstrong et de D.E. Lowe, cf. M. Hicks, Art. Woodville [Wydeville], Anthony, second Earl Rivers, Oxford Dictionary of National Biography, t. 60, p. 224-226 ; ID., Art. Anthony Wydeville, Who's Who in Late Medieval England, Londres, 1991, p. 346-348. 
Pizan et le Cordiale de quattuor novissimis de Gerard van der Vlyderhoven, dans sa version française (Traité des quatre dernières choses, trad. Jean Miélot, 1455), éditée à Bruges en 1476 par Caxton et Colard Mansion. Chacune des œuvres de Rivers sera précisément imprimée par Caxton, les Dictes constituant d'ailleurs le premier livre imprimé en Angleterre, en 1477 (Proverbes en 1478 et Cordyal en 1479) ${ }^{25}$. Caxton signale encore quelques œuvres perdues, en particulier des ballades relatives aux sept péchés capitaux, mais les seuls vers de Woodville à être parvenus jusqu'à nous portent sur l'inconstance de la Fortune. Le comte Rivers eut des rapports avec la Renaissance italienne et ses choix littéraires furent approuvés par les humanistes. Autre faîte dans la carrière chevaleresque de Woodville, une joute, fameuse entre toutes. Alors qu'une ébauche d'union entre Charles de Charolais et Anne d'York, la sœur d'Édouard IV, la fille de Richard d'York et de Cécile Neville, avait avorté en 1454, Philippe le Bon s'y étant obstinément opposé, le projet est de nouveau à l'ordre du jour dans les années soixante, à propos cette fois de Marguerite, autre sœur du souverain anglais, et pour peu qu'il se double de dispositions économiques et militaires. Dès mars 1466, après une reprise de contact entre Charolais et Édouard IV fin 1464 ou début 1465, des discussions sont engagées entre les délégations anglaises et bourguignonnes ; elles déboucheront sur des accords commerciaux et politiques, le 23 octobre notamment. Parmi les Bourguignons, Louis, seigneur de Gruuthuse, et le demi-frère de Charolais, Antoine, Grand Bâtard de Bourgogne. Ce dernier prendra une part prépondérante aux négociations qui devaient aboutir au mariage de Charles et de Marguerite d'York, tout particulièrement au cours de ce voyage qu'il fit en Angleterre à la mi-1467. L'on sait qu'il en ira de même pour Anthony Woodville. Bien que sans doute pur prétexte à des discussions politiques discrètes, le sommet curial de ce déplacement bourguignon sera sans conteste une joute opposant, en juin, à Londres, à l'emplacement de Smithfield et dans la magnificence la plus absolue, Antoine de Bourgogne à Anthony Woodville, le champion des dames anglaises. Mais les joutes tournent court, car une triste nouvelle tombe: Philippe le Bon vient de

25. Sur Caxton et les aspects littéraires de la vie d'Anthony Woodville, cf. notamment N.F. BLAKE, Caxton : England's First Publisher, Londres, 1976, p. 45-47, 109110,179, 192-193 et passim ;H.S. BENNETT, English Books and Readers, 1475 to 1557, beeing a Study in the History of the Book Trade from Caxton to the Incorporation of the Stationers' Company, 2e éd., Cambridge, 1970, p. 12, 293, 295, 316 ; R. DeAcon, A Biography of William Caxton, the First English Editor, Printer, Merchant and Translator, Londres, 1976, p. 58-60, 118-122, 133; ANTHONY WoOdVILLE, EARL Rivers, The Cordyal, edited from M 38 A1, The Museum Meermanno Westreenianum, The Hague, par J.A. Mulders, Nimègue, 1962 (éd. de la trad. Woodville d'après la $1^{\text {re }}$ éd. Caxton de 1479). Dans ces divers ouvrages, les titres des traductions réalisées par Woodville présentent des divergences. Voir encore N.F. BLAKE, Art. Caxton, William, Oxford Dictionary of National Biography, t. 10, p. 694-698 (et bibl.). 
mourir ${ }^{26}$. C'est très exactement ce fait impressionnant que retiendra Jean de Wavrin dans sa chronique, un Jean de Wavrin, précisément, qui avait fait le voyage vers l'Angleterre en compagnie du Grand Bâtard ${ }^{27}$. Si tant est que le lignage des Woodville fut pour beaucoup dans l'émergence de la culture bourguignonne à la cour d'Angleterre $\mathrm{d}^{28}$ - elle devrait, par exemple, au comte Rivers la réintroduction, en son sein et dans l'esprit bourguignon, des tournois romantiques, oubliés sous les Lancastre-, il ne semble pas exagérément audacieux de penser qu'une certaine connivence, un peu à la manière de celle qui exista entre La Marche et William Lord Hastings, ami et soutien très proche d'Édouard IV, lui aussi grand amateur de manuscrits, a pu naître entre un Anthony Woodville, appelé à s'ouvrir à la richesse des lettres, et ce chroniqueur qu'Antoine de Bourgogne, son émule des joutes de juin 1467, avait en quelque sorte emmené dans ses bagages, une complicité dont a pu bénéficier la cour de Londres. Cependant, il est aussi au moins deux autres réalités qui, un peu plus tard peut-être, ont pu permettre la diffusion des richesses culturelles bourguignonnes en terre anglaise et, très concrètement, offrir quelque rayonnement au Bâtard de Wavrin. Il n'est évidemment pas nécessaire de s'étendre ici outre mesure sur les retombées qu'eut fatalement, en ces matières, le mariage du duc Charles et de Marguerite d'York en 1468. En revanche, l'exil d'Édouard IV, entre octobre 1470 et mars 1471, mérite quelques mots ${ }^{29}$. Accompagné notamment du comte Rivers, il trouva refuge à Bruges auprès de Louis de Gruuthuse, l'un des plus grands bibliophiles bourguignons, lequel, le fait n'est peut-être pas innocent et mérite d'être signalé, semble avoir possédé un manuscrit ayant appartenu à Jacquette de

26. Sur les négociations en vue de l'union de Charles et de Marguerite, sur la joute et le mariage lui-même, cf. M.R. Thielemans, Bourgogne et Angleterre. Relations politiques et économiques entre les Pays-Bas bourguignons et l'Angleterre, 1435-1467, Bruxelles, 1966, p. 415, 418-424 ; Fr. Jeuris, Antoine, Grand Bâtard de Bourgogne, Mém. de Lic. en Histoire, Université de Liège, 2000-2001, p. 97-102 ; A. CommIES, Nul ne s'y frote. Een biografische schets van Anton, Bastaard van Bourgondië, Excursiones mediaevales. Opstellen aangeboden aan Prof. Dr. A.G. Jongkees door zijn leerlingen, Groningue, 1979, p. 65-68 ; H. Dubors, Charles le Téméraire, Paris, 2004, p. 137-138, 181196 ; ARMStRONG, L'échange culturel entre les cours d'Angleterre et de Bourgogne, p. 404-407 ; S. ANGLO, Anglo-Burgundian feats of arms : Smithfield, June 1467, Guildhall Miscellany, t. 2, n 7, 1965, p. 271-283.

27. J. DE WAVRIN, Recueil des croniques et anchiennes istories de la Grant Bretaigne, éd. HARDY, t. 5, p. 542-543.

28. Sur tout ce qui suit, cf. ARMSTRONG, L'échange culturel entre les cours d'Angleterre et de Bourgogne, p. 404-409, 414-415.

29. Ross, Edward IV, p. 153, 159-160 et, surtout, L. VISSER-Fuchs, Il n'a plus lion ne lieppart, qui voeulle tenir de sa part: Edward IV in Exile, October 1470 to March 1471, L'Angleterre et les pays bourguignons : relations et comparaisons ( $X V^{e}-X V I^{e}$ siècles), éd. J.M. Cauchies, Publication du Centre européen d'Études bourguignonnes (XIVe-XVI ${ }^{e}$ s.), t. 35, 1995, p. 91-106. 
Luxembourg puis à son fils, Anthony Woodville, et donc ayant peut-être été offert par ce dernier à celui qui l'accueillit en terre flamande ${ }^{30}$. Sachant que l'un des manuscrits de la chronique de Jean de Wavrin, le seul complet, celuilà même qui fut utilisé par les éditeurs de l'œuvre, au XIX', à savoir l'actuel B.N.F. fr. 74-85, datant approximativement de 1484, appartenait à Louis de Gruuthuse avant de passer dans les collections du roi Louis XII à Blois, l'on ne peut guère s'empêcher de penser que le roi d'Angleterre a eu aussi toute latitude pour rencontrer chez son hôte brugeois celui qui allait bientôt lui dédicacer un exemplaire de son œuvre ${ }^{31}$. L'on a d'ailleurs parfois vu dans la miniature appartenant au manuscrit offert à Édouard IV, où Wavrin présente sa chronique au roi et où apparaîtraient également, à l'avant plan, les ducs de Clarence et de Gloucester, ainsi qu'en marge, le héraut de l'ordre de la Jarretière, John Smert, un épisode de l'exil brugeois du souverain anglais ${ }^{32}$.

Passons à présent à la dernière partie de cette étude : les années antérieures à 1471 et la guerre des Deux Roses, telles qu'elles sont exposées par Wavrin,

30. Il s'agit du ms. LONDREs, British Library, Harley 4431, soit les Proverbes moraux de Christine de Pizan dont Anthony Woodville réalisa la traduction en anglais. Il fut enluminé par le Maître de la Cité des Dames. Reproduction dans J. BACKHOUSE, The Illuminated Page. Ten Centuries of Manuscript Painting in the British Library, Londres, 1997, p. 158-159. Sur Gruuthuse, principalement mécène, cf. M.P.J. MARTENS et AL., Lodewijk van Gruuthuse. Mecenas en europees diplomaat ca 1427-1492, Bruges, 1992 ; L. VAn Praet, Recherches sur Louis de Bruges, seigneur de la Gruthuyse, Paris, 1831 ; H. WiJsman, Gebonden Weelde. Productie van geïllustreerde handschriften en adellijk boekenbezit in de Bourgondische Nederlanden (1400-1550), Leyde, 2003, p. 240-248; M. VALE, An Anglo-Burgundian Nobleman and Art Patron : Louis de Bruges, Lord of la Gruthuyse and Earl of Winchester, England and the Low Countries in the Late Middle Ages, éd. C. Baron et N. SAul, Stroud-New York, 1995, p. 115-131 ; Armstrong, L'échange culturel entre les cours d'Angleterre et de Bourgogne, p. 410-412.

31. J. DE WAVRIN, Recueil des croniques et anchiennes istories de la Grant Bretaigne, éd. HARDY, t. 1, p. CCXI-CCXIII ; ID., Anchiennes chronicques d'Engleterre, éd. Dupont, t. 1, p. III, VIII n. 2 ; MARTENS, De Librije van Lodewijk van Gruuthuse, Lodewijk van Gruuthuse. Mecenas en Europees diplomaat, p. 121, 147 ; NABER, Manuscrits d'un bibliophile bourguignon, p. 23-24. L'œuvre a été enluminée par le Maître de la Chronique d'Angleterre, artiste qui s'inspira de Loyset Liédet; il se reconnaît aisément à ses peintures en vue plongeante, truffées de personnages, dont les vêtements, arbres et architectures sont exécutées de façon sommaire, et à ses scènes nocturnes, exceptionnelles pour l'époque (M. SMEYERS, L'art de la miniature flamande du VIII a au XVI e siècle, Tournai, 1998, p. 362-363, qui situe le ms. vers 1470).

32. LondREs, British Library, ms. Royal. 15.E.IV, fol. $14 \mathrm{r}^{\circ}$ (cf. ill. infra, p. 527). Cf. légende de ladite miniature, qui orne la jaquette de ARMSTRONG, L'échange culturel entre les cours d'Angleterre et de Bourgogne. Voir aussi n. 55. Sur la bibliothèque royale anglaise sous Édouard IV, cf. J. BACKHOUSE, Founders of the Royal Library : Edward IV and Henry VII as Collectors of Illuminated Manuscripts, England in the Fifteenth Century. Proceedings of the 1986 Harlaxton Symposium, Woodbridge, 1987, p. 23-41 et ill. Cf. aussi KEREWICH, Edward IV, William Caxton, and literary Patronage. 
par les principaux chroniqueurs qui lui sont contemporains, et, plus important sans doute, la perception qu'a pu en avoir la société bourguignonne du temps.

De la Chronique de Mathieu d'Escouchy, qui s'étend pourtant jusqu'en 1461, année au cours de laquelle Édouard IV monte sur le trône d'Angleterre, la guerre des Deux Roses est quasiment absente. Ni le premier souverain York, ni même Warwick ne se trouvent mentionnés dans cette œuvre. Quelques propos seulement, d'ailleurs assez vagues voire erronés, y sont consacrés aux aspirations à la couronne de Richard d'Y ork $^{33}$. Il n'y a aussi que très peu à retenir d'Olivier de la Marche et de ses Mémoires quant au sujet qui nous occupe ici. Inscrits dans son second livre et formés de notes prises au cours des événements, ses propos sur la guerre des Roses se limitent à une brève et lâche évocation, sans grande erreur mais aussi sans véritable originalité, des démêlés du roi avec le duo formé de Clarence et surtout de Warwick, dans les années 1469-1471 $1^{34}$. Il est par ailleurs bien plus loquace dès lors qu'il s'agit d'exposer le déroulement de la joute de 1467, assurément davantage pour ses implications bourguignonnes qu'insulaires ${ }^{35}$. L'on remarquera cependant qu'Henri VI lui apparaît comme un homme simple d'esprit, laissant tout le pouvoir à une épouse sachant et de grant esprit ${ }^{36}$. Vision des événements bien superficielle également chez le quasi-contemporain de La Marche, Jean de Haynin, un guerrier dont, il est vrai, on le sait, la spécialité est surtout le récit de bataille croqué sur le vif et mis au net peu après. Édouard IV et Warwick finirent par se brouiller après avoir gouverné de concert. Les causes pour quoy bonnement je ne saroie dirre, nous précise l'auteur, qui accède à une partie de l'information, certes, mais parce que renoumée couru ${ }^{37}$. Une véritable originalité, tout de même : 1 'insertion dans le cours du récit d'une lettre, non datée, mais probablement d'avril 1471, adressée par Marguerite d'York à sa belle-mère Isabelle de Portugal, véritable compte rendu des combats menés dans l'Angleterre de 1471, une « newsletter » en d'autres termes ${ }^{38}$. Plus intéressant pour le chercheur apparaît le texte offert par Jacques du Clercq dans ses Mémoires. À dire vrai, la

33. M. D'Escouchy, Chronique, éd. Du Fresne de Beaucourt, t. 1, p. 298-303 ; t. 2, p. $113,261-262,353$.

34. O. de la Marche, Mémoires, éd. Beaune et D'Arbaumont, t. 3, p. 68-70, 236-237.

35. Ibid., t. 3, p. 41, 48-57. Hormis chez Wavrin, c'est bien évidemment l'Angleterre en ce qu'elle a comme incidence sur l'histoire française et bourguignonne qui intéresse prioritairement les chroniqueurs.

36. Ibid., t. 2, p. 209.

37. J. De Haynin, Mémoires, éd. Brouwers, t. 2, p. 91.

38. Ibid., p. 125-129. Le document est également édité dans L.P. GACHARD, Analectes historiques, $3^{\mathrm{e}}$ sér., Bulletin de la Commission royale d'Histoire, $2^{\mathrm{e}}$ sér., t. 7 , 1855, p. 47-52 et dans J. De WAVRin, Anchiennes chronicques d'Engleterre, éd. Dupont, t. 3, p. 210-215. 
valeur de son propos ne réside pas vraiment dans les faits qu'il relate, car, si le mémorialiste consacre des chapitres entiers, mais brefs, aux événements qui secouèrent l'Angleterre avant 1467, date à laquelle s'achève son œuvre, et si son évocation des faits est plus étoffée que chez La Marche, par exemple, en particulier lorsqu'il est question de la reine d'Angleterre Marguerite d'Anjou, son récit n'en reste pas moins assez aérien. Toutefois, pour peu que l'on se penche sur le récit composé à propos des événements qui précédèrent l'arrivée au pouvoir d'Édouard IV, non pas celui qui est inséré dans le continuum des Mémoires, peu disert sur la question, mais dans une narration ajoutée en appendice ${ }^{39}$ et précédée d'une préface dans laquelle l'auteur nous précise que après ce que je [...] ay clos mon premier volume des choses advenues en mon temps et venues à ma connaissance[...] j'ai esté adverti et sceu le commenchement des divisions et les causes dont elles meurent audit royaulme, l'on constate sans difficulté, par le choix des sujets, par l'articulation du texte, par l'utilisation d'expressions particulières, par certaines indications chiffrées, que les exposés de Du Clercq et de Wavrin sont très strictement apparentés, le premier produisant une version allégée du récit du second ou, processus inverse, Wavrin amplifiant Jacques du Clercq ${ }^{40}$. J. Stengers, dans l'étude qu'il a consacrée à la parenté entre diverses œuvres bourguignonnes, dont celles de Wavrin et de Jacques du Clercq ${ }^{41}$, a exposé les raisons pour lesquelles la seconde hypothèse doit être retenue. Reste que les propos tenus dans la préface évoquée plus haut semblent dire le contraire...

Hauteur, élévation par rapport aux faits et aux personnages, voilà la qualité première qu'affichent les récits de Chastelain et de Commynes, notamment dès lors qu'il est question de la guerre des Deux Roses. Du premier, je ne souhaite pas parler longuement, car il ne m'est guère possible d'intégrer ici la matière de son Temple de Bocace, cette consolation pour

39. J. du Clerce, Mémoires, éd. ReIfFenderg, $2^{\mathrm{e}}$ éd., t. 1, p. 435-451 (citation infra, p. 435).

40. Exemple : ibid., t. 1, p. 442-443 : après lequel son partement il revint jusques à cinq milles près de Londres, et avecq lui et en sa compagnie, les comte de Binsbre et $S^{r}$ de Kohan, avecq bien vingt mille combattants, pour lesquels combattre le roy wida de Londres, avecq lui le duc de Presse, le duc de Norffort, le duc de Benguignam, et le duc de Sombreset, et des aultres sieurs, desquels la plus grande partie aimoient bien leducd'Yorck [...] ; J. DE WAVRIN, Recueil des croniques et anchiennes istories de la Grant Bretaigne, éd. HARDY, t. 5, p. 265 : si se party de Londres, mais assez tost aprez il sen vint logier a chincq milles prez de Londres, en sa compaignie le comte de Vinchier et le seigneur de Cophain, en sa routte bien vingt mille bons combatans; si se deliberent le roy Henry et son conseil de le aller combattre adcompaignie des ducs dExces, de Noffocz, de Bouquinghuem et de plusieurs grans seigneurs et barons quy amoient bien le duc dYork [...]. Comparer également J. DU CLERCQ, Mémoires, t. 1, p. 444445 et J. DE WAVRIN, Recueil des croniques et anchiennes istories de la Grant Bretaigne, éd. HARDY, t. 5, p. 268-269.

41. Stengers, Sur trois chroniqueurs, p. 129. 
Marguerite d'Anjou, l'épouse de l'infortuné Henri VI d'Angleterre ${ }^{42}$. Je signalerai cependant que la guerre des Deux Roses et, plus largement, l'histoire et le peuple anglais n'ont qu'une importance secondaire chez Chastelain - indiciaire de Bourgogne et, à ce titre, intéressé par les Anglais et les événements anglais dès lors qu'ils ont quelque incidence sur le vécu bourguignon - hormis dans les deux premiers livres de sa chronique qui ne nous concernent guère ici. Ainsi, alors qu'en1470, Henri VI vient de recouvrer son trône, ne déclare-t-il pas : Je n'ai point fait fortes enquestes de ceste matière, ne comment le comte de Warwyc se maintint en Londres, ne comment Edouard se mit sus et se prepara a la deffense de son royaume contreluy, [car peu m'en aétéet est de faveur, et peu y attens de louenge ou de mérite, par y avoir fort laboré $]^{43}$ ? Restent une trame assez générale et des personnages sur lesquels il a bien sûr une opinion. Marguerite a été expulsée de son royaume et déshéritée avec son époux, tandis qu'Édouard IV, du sang d'York, estoit parvenu à la couronne par violence, et en avoit débouté l'héritier proxisme du sang de Lancaste ${ }^{44}$, de sorte qu'il estime bien téméraire de vouloir s'acoquiner avec pareil individu. Au bout du compte, ces individualités historiques et princières serviront sa vision résolument péjorative des Anglais qui fiers sont et mal traitables en leur fumier $^{45}$, et qui, à l'image de Warwick, ne s'élèvent pas au-delà du porc-sengler sauvage, de nation et extraction inhumaine ${ }^{46}$. Chez Commynes, à présent, le récit consacré à la guerre des Deux Roses, globalement limité, pour la période qui nous occupe, aux fuites et exils de Warwick et d'Édouard IV et à leurs retours successifs en Angleterre ${ }^{47}$, est bien moins précis, moins riche en personnages, en faits d'armes, en un mot en détails - il déclare dire grossement ce qu'il a vu, su et entendu dire par les princes ${ }^{48}$, , que celui, on va le voir, du Bâtard, même si l'épisode mettant en scène, de façon très circonstanciée, Wenlock, le lieutenant de Warwick à Calais, champion, comme le Téméraire d'ailleurs, de la palinodie politique, apparait plutôt original ${ }^{49}$. Toutefois, comme chez Chastelain, tout ce que dit Commynes à propos des Roses, événements, personnages, enchaînement des faits sera, comme l'a parfaitement montré J. Dufournet ${ }^{50}$, prétexte à l'exposé de jugements très nuancés sur le pouvoir,

42. G. Chastelain, Le Temple de Bocace, éd. Bliggenstorfer. L'analyse de cette œuvre, dans la perspective qui est la nôtre, a d'ailleurs été ébauchée par DouDET, De l'allié à l'ennemi, p. 90-93. Plus largement, sur Chastelain et les Anglais, cf. Delclos, Témoignage de Georges Chastellain, p. 245-261.

43. G. Chastelain, CEuvres, éd. Kervyn de Lettenhove, t. 5, p. 489.

44. Ibid, t. 4 , p. 158.

45. Ibid, t. 3, p. 338.

46. Ibid, t. 5, p. 495.

47. Ph. De Commynes, Mémoires, éd. Calmette, t. 1, p. 190-217.

48. Ibid., p. 190.

49. Ibid., p. 194-196, 198-199, 206.

50. J. Dufournet, Philippe de Commynes et l'Angleterre, dans ID., Philippe de 
la politique, sur ceux qui les incarnent et, in fine, sur le genre humain ${ }^{51}$... À cet égard, des plus éclairants est le portrait que Commynes brosse d'Édouard IV, portrait tout en finesse, bien moins binaire que chez Wavrin : ce bel homme ne manquait pas de qualités, de courage notamment, mais fut aussi en quelque sorte un usurpateur, un dissimulateur, un indolent, crédule, imprévoyant, irraisonnable et dilettante, qui a abusé des plaisirs de l'amour et de la table et qui mourra honteux et malheureux ; il s'impose alors comme un raccourci partiel, mais éclairant, du peuple anglais, du prince en général et de l'humanité tout entière ${ }^{52}$.

Wavrin, maintenant. Il passe pour l'un des rares chroniqueurs continentaux $^{53}$ à proposer une contribution substantielle à la connaissance de la guerre des Deux Roses ; de fait, l'essentiel de son volume six est consacré au conflit qui mit aux prises les tenants respectifs des roses rouge et blanche, les Lancastre et les York, un conflit pour lequel, faut-il le préciser, Wavrin fait de l'historiographie d'actualité, fondée, à côté des "newsletters », sur des rencontres, des conversations, des expériences personnelles, même si l'on $n^{\prime} y$ rencontre pas une de ces expressions dont il a d'ordinaire plaisir à truffer son texte, à savoir je acteur de ceste euvre vey à mes yeulx ou moy acteur estant present $^{54}$. On le reconnaîtra aisément, par sa longueur, par son enchevêtrement, par son foisonnement de faits, de lieux, de personnages, d'événements, le récit de la guerre des Deux Roses qu'a rédigé Wavrin est d'une richesse à nulle autre pareille ${ }^{55}$, que les historiens anglo-saxons ne peuvent

Commynes. Un historien à l'aube des temps modernes, p. 114-128 (reprod. de ID., op. cit., Bulletin de la Société d'Histoire de Comines-Warneton et de la Région, t. 21, 1991, p. 103118).

51. Édouard IV fut de ces dirigeants qui ne tiennent pas compte des conseils qui leur étaient donnés, que Dieu confronta à des ennemis inattendus, car ils n'étaient plus conscients de leur félicité politique ; les rencontres entre chefs d'États génèrent tellement d'occasions de frictions qu'il vaudrait mieux les éviter... : Ph. DE COMMYNES, Mémoires, t. 1, p. 54, 78-79, 141, 204-205, 245-247, 299-300.

52. Ibid., t. 1, p. 197, 200, 203 ; t. 2, p. 8-9, 37, 60, 64, 76-77, 230, 239-240, 245-246, 304, 332-334 et passim.

53. GransDEn, Historical Writing in England, t. 2, p. 288-301; НicKs, Edward IV, p. 29, qui, outre Wavrin et Commynes (infra), mentionne deux œuvres non bourguignonnes, celles de Thomas Basin et de Jean de Roye.

54. J. DE WAVRIN, Recueil des croniques et anchiennes istories de la Grant Bretaigne, éd. HARDY, par exemple, t. 2, p. 229 (Azincourt, citation) ; t. 3, p. 70, 101, 109, 303 (Beaugency, citation).

55. Il conviendrait assurément de comparer, point par point, la relation de Wavrin à celles que proposent les autres chroniqueurs, anglais pour la plupart, tâche qui, à n'en pas douter, pourrait donner lieu à tout un ouvrage, tout comme d'ailleurs la comparaison de la matière bourguignonne proposée par Wavrin avec le récit de ses homologues. 
en aucun cas négliger. Il est, on l'aura compris, le seul à proposer, dans l'espace bourguignon un véritable reportage journalistique, presque au jour le jour, d'un conflit civil d'importance. Il apparaît aussi quasiment comme le seul média ayant éventuellement permis aux structures élevées de la société bourguignonne de prendre connaissance d'un ensemble d'événements d'une complexité considérable, qui ne furent pas sans influer sur l'histoire bourguignonne elle-même, et ce d'autant plus que l'œuvre semble avoir connu une vraie diffusion, si l'on en croit le nombre passablement élevé de manuscrits conservés ${ }^{56}$. Au-delà de ces constats, le récit de Wavrin laisse diverses impressions. Force est de constater, tout d'abord, qu'il n'a pas toujours la précision que l'on était en droit d'attendre d'un texte très étoffé, très touffu et généralement bien informé. Telle est la conclusion à laquelle on aboutit, par exemple, lorsque l'on analyse sa description des années 14501461, le premier millésime correspondant au début du conflit entre Edmund Beaufort, duc de Somerset, favori d'Henri VI et surtout de la reine Marguerite d'Anjou, et Richard, duc d'York, le père du futur Édouard IV, ce dernier

56. Le recensement (exhaustif ?) de ceux-ci a été réalisé : VISSER-Fuchs, Pour recreer les esperitz. Dans la foulée, l'étude des peintures enluminées et des rapports texte/image serait des plus appréciables. L'on mentionnera, d'ores et déjà, outre les mss mentionnés plus haut et en en tenant compte dans le comptage des tomes (LONDREs, British Library, mss Royal. 14. et 15.E.IV, t. 3 et 1 - reproduction de ce ms. dans BACKHOUSE, The Illuminated Page, p. 195-196, qui date le ms. des années 1470 ; PARIS, B.N.F., ms. fr. 74-85, complet), les volumes suivants : pour le t. 1 (5 exemplaires), B.N.F., mss fr. 71 et 2807, VIENNE, Österreichische Nationalbibliothek, Cod. 2534 [daté de vers 1470, frontispice attribué au maître d'Antoine de Bourgogne, les autres enluminures à celui de la Chronique d'Angleterre, déjà évoqué (SMEYERS, L'art de la miniature flamande, p. 363, qui place le ms. vers 1470] ; pour le t. 2 (4 exemplaires), B.N.F., mss fr. 72 et 87, SAN MARINO (U.S.A.), Huntington Library, HM 28562, OxFORD, Bodleian Library, Laud. Misc. 653, et, partiellement, LA HAYE, Koninklijke Bibliotheek, 133 A 7 I (également évoqué par NABER, Manuscrits d'un bibliophile bourguignon, p. 24 n. 3); pour le t. 3 (3 exemplaires), LA HAYE, Koninklijke Bibliotheek, 133 A 7 II ; pour le t. 4 (3 exemplaires), BALTiMORE, Walters Art Museum, Ms. W. 201 (L.M.C. Randall et Al., Medieval and Renaissance Manuscripts in the Walters Art Gallery, t. 3, Belgium, 1250-1530, vol. 2, Baltimore-Londres, 1997, p.387-392, n² 276, et ill., p. 561 ; Medieval Mastery. Book Illumination from Charlemagne to Charles the Bold, 800-1475, Turnhout-Louvain, 2002, p. 316-317), B.N.F., ms. fr. 23058 ; pour le t. 5 (2 exemplaires): LA HAYE, Koninklijke Bibliotheek, 133 A 7 III et des fragments dans B.N.F., ms. fr. 23058 ; pour le t. 6, fragments dans B.N.F., mss fr. 23058, 23059 et 15491. Parmi les dédicataires des manuscrits signalés, l'on mentionnera, outre Édouard IV et Louis de Gruuthuse : Pietro et Claudio de Villa, des Lombards ; Jacques d'Armagnac, duc de Nemours ; Englebert II ou son neveu, Henri III de Nassau. Dans la même ligne d'idées, nous n'avons pas estimé devoir tenir compte ici de A. DE BUT, Chronique, éd. KeRvYN DE LeTtenhove, où il est pourtant question des événements anglais, compte tenu du peu de diffusion globale de l'œuvre (cf. LAMBERT, Chronicles of Flanders, p. 120-130). 
ceignant la couronne d'Angleterre au cours de la seconde année. L'on constate en effet que Wavrin se montre assez précis dès lors qu'il s'agit de décrire préparatifs, dispositifs et expéditions militaires. Les deux batailles de Saint-Albans, celles de Blore Heath, Northampton, Wakefield, Towton, d'autres encore, plus tard, seront ainsi exposées dans toutes leurs dimensions $^{57}$. Il en va de même lorsque Wavrin évoque le parcours géographique, politique et militaire des beau-frère et neveu de Richard d'York, les deux Richard Neville, comtes de Salisbury et de Warwick, et en particulier les relations étroites entre ce dernier et la ville de Calais dont il assumait le commandement $^{58}$. En revanche, pour ce qui est de l'enchevêtrement des événements politiques que connaît la période concernée, s'il lui arrive parfois d'y réfléchir, de tenter de l'expliquer, voire de porter un jugement sur lui - lorsqu'il s'interroge sur les aléas de la succession au trône d'Angleterre, sur le pourquoi du mariage d'Édouard et d'Élisabeth Woodville, par exemple $^{59}-$, il lui échappe aussi parfois dans une certaine mesure, en ce compris des faits d'une extrême importance. L'on constate par exemple, toujours pour la période 1450-1461, qu'il n'évoque aucunement les crises d'hébétude qui frappent Henri VI, la bâtardise dont on affuble son fils, le prince de Galles, bâtardise, on le sait, qu'excipèrent les York pour se substituer au Lancastre, mais aussi des faits politiques majeurs comme le pardon général accordé par le roi à ses opposants, en 1452, ou le premier protectorat de Richard d'York alors qu'Henri VI était empêché6 ${ }^{60}$. Une autre spécificité du récit que fait Wavrin de la guerre des Deux Roses est liée à mon sens aux options politiques adoptées par le chroniqueur, à toute la conviction de celui qui est aussi un homme de cour bourguignon. Incontestablement, élaboré alors que les relations entre les Valois de Bourgogne et les York étaient à peu près

57. J. DE WAVRIN, Recueil des croniques et anchiennes istories de la Grant Bretaigne, éd. HARDY, par exemple, t. 5, p. 267-268, 319-326, 336-341. Saint-Albans (22 mai 1455), Blore Heath (23 septembre 1459), Northampton (10 juillet 1460), Wakefield (30 décembre 1460), Saint-Albans (17 février 1461) et Towton (29 mars 1461) sont de célèbres rencontres armées de la guerre des Deux Roses. Sur ces événements, cf. A. Goodman, The Wars of the Roses. Military Activity and English Society, 1452-97, rééd., Londres, New York, 1991, p. 23-24, 27-28, 37-39, 42-43, 46-48, 51-52. Evocation précise de la période dans P.A. JoHnson, Duke Richard of York, 1411-1460, Oxford, 1988, p. 155224 ; Ross, Edward IV, p. 18-63.

58. J. DE WAVRIN, Recueil des croniques et anchiennes istories de la Grant Bretaigne, éd. HARDY, par exemple, t. 5, p. 267 s. Sur Warwick et Calais, dans et hors de la période évoquée ici, cf. M. Hicks, Warwick the Kingmaker, Oxford-Malden (Mass.), 1998.

59. J. DE WAVRIN, Recueil des croniques et anchiennes istories de la Grant Bretaigne, éd. HARDY, par exemple, t. 5, p. 343, 352, 455-456.

60. Sur ces faits, cf. B. Wollfe, Henry VI, rééd., New Haven-Londres, 2001, p. 1819, 253-256, 270-273, 280-285 ;H.E. MAURER, Margaret of Anjou. Queenship and Power in Late Medieval England, Woodbridge, 2003, p. 45-48 ; R.A. GRIFrITHs, The Reign of King Henry VI, rééd., Phoenix Mill, Thrupp, Stroud, 1998, p. 693-700 et n., 715-738 et n. 
harmonieuses, le sixième volume du Recueil reflète cette connivence du moment: yorkiste, la description que fait Wavrin de l'antagonisme entre Lancastre et York l'est quasi de bout en bout. Aussi cette évidence amène-telle le chroniqueur à brosser des portraits parfois quelque peu monolithiques des divers protagonistes du conflit. De Marguerite d'Anjou, liée à la maison de France, car fille du roi René, duc d'Anjou, de Lorraine et de Bar, prétendant au trône de Naples, Wavrin n'est assurément pas le sigisbée. Certes, les critiques sont rarement outrancières, mais le propos n'est que réprobation. Henri VI, dans l'armée duquel Wavrin a combattu, échappe quant à lui à l'anathème du chroniqueur. Le souverain Lancastre, qui, nous dit Wavrin, ne sait jamais rien de rien, apparaît comme un pantin entre les mains d'une Marguerite d'Anjou qui le mène en galère ${ }^{61}$. D'Édouard IV, il est brossé un portrait impressionniste, par touches successives. Là encore, rien d'outrancier, mais absolument rien non plus de négatif. Reste Warwick, sorte de Janus, loué pour ses qualités puis, même si une certaine retenue reste de mise, résolument critiqué dès que sa fidélité au roi devient chancelante ${ }^{62}$. Ces portraits, dont la teneur est indissolublement liée aux options politiques du chroniqueur, seront fatalement univoques, ce qui, sans doute, contribua à lui interdire de gagner de la hauteur, en terme de jugement notamment, par rapport à son sujet.

Que conclure de cette analyse largement à poursuivre, dont le dessein principal était d'esquisser quelques pistes de réflexion? Jean de Wavrin, chroniqueur sous les ducs Philippe et Charles de Bourgogne, est l'auteur d'une Histoire d'Angleterre, dont l'ultime partie témoigne d'une nette prédilection, de sa part, pour le régime yorkiste et fatalement celui qui en est l'incarnation, le roi Édouard IV. L'on peutémettre l'hypothèse selon laquelle le frère de la reine d'Angleterre, Anthony Woodville, a pu servir de trait d'union entre le souverain anglais et le chroniqueur bourguignon, notamment à l'occasion de la joute qui mit aux prises, en 1467, sur le sol anglais, ledit Anthony et le Grand Bâtard Antoine de Bourgogne, qui, pour la circonstance, se trouvait accompagné de Wavrin. J'ai par ailleurs envie de dire qu'incontestablement, si l'on prend uniquement en compte l'œuvre des chroni-

61. J. DE WAVRIN, Recueil des croniques et anchiennes istories de la Grant Bretaigne, éd. HARDY, par exemple, t. 5, p. 268 : la royne, qui moult heoit le duc dYorc pour la mort du duc de Sombresset, fist tant devers le roy, quelle menoit a son plasir, [...] que le duc dYorc fut du tout deboutte du gouvernement et auctorite quil avoit ou royaulme AAngleterre; p. 283 : maistre Ormont, le mignon de la royne dAngleterre ; p. 327 : la royne Margueritte, qui estoit soubtille et mallicieuse.

62. Fine analyse du personnage vu par Wavrin, dans VISSER-FuCHS, « Warwick, by himself $»$. 
queurs ${ }^{63}, c^{\prime}$ est pour l'essentiel chez Wavrin - et dans une moindre mesure, chez Commynes, qui ne le souhaitait pas, et chez Du Clercq - que ses contemporains, tout au moins les princes et les nobles qui, par les nombreuses copies qui en furent faites, avaient accès à son œuvre, pouvaient aller puiser la matière susceptible de parfaire leur connaissance du détail des événements qui s'étaient déroulés Outre-Manche entre 1450 et 1471, de venir s'ajouter à ce que l'on pouvait savoir grâce aux conversations entre vifs et à la circulation des nouvelles, notamment dans le cadre des échanges diplomatiques. Ce faisant, c'est certes à un récit luxuriant, affichant une débauche de détails, mais aussi à un spectacle assez orienté de l'histoire que Wavrin les conviait. Au vrai, comment ne pas regretter de ne pouvoir lire les pages que Wavrin n'aurait pas pu ne pas écrire, mais qu'il n'eut pas la possibilité de rédiger, rejoint qu'il fut entre-temps par la mort, à propos du traité de Picquigny de 1475, en d'autres termes de la trahison qui fut celle d'Édouard IV à l'égard de Charles le Téméraire ? Peut-être celui dont l'aptitude à s'adapter aux aléas de la politique était excellente eût-il été forcé de reconsidérer quelque peu la belle œuvre de " propagande » yorkiste dont il avait fait le sixième volume de son Recueil.

$$
\text { F.N.R.S. - Université de Liège }
$$

Alain MARChANDISSE

63. Cf. cependant, pour un autre type de source (d'informations), l'article très circonstancié de ID., Edward IV's « memoir on paper», notamment la liste des mss renfermant celui-ci et son édition. 


\section{DROITS ÉLECTRONIQUES RÉSERVÉS SUR IMAGE(S)}

Jean de Wavrin offre son œuvre à Édouard IV.

JEAN DE WAVRIn, Recueil des croniques et anchiennes istories de la Grant Bretaigne, années 1470, British Library, ms. Royal. 15.E.IV, fol. 14 r $^{\circ}$

(C B.L., By permission of the British Library) 